\title{
¿Puede hablarse de un cogito deleuziano? Una interpretación de las síntesis pasivas de Diferencia y repetición
}

\section{Can we speak of a Deleuzian cogito? An interpretation of Difference and repetition's passive synthesis}

\section{Diego Abadi*}

Resumen: Dentro de las relaciones libres y discordantes que establecen entre ellas las facultades, Deleuze destaca una experiencia particular, la de la génesis del pensamiento, en la que las facultades mantienen sin embargo una ordenación fija: el pensamiento tiene siempre su origen en la sensibilidad, de allí se comunica a la imaginación, de ésta a la memoria y a continuación al pensamiento. $\mathrm{Y}$ es gracias a esta fijeza que dicha experiencia puede ser identificada y nombrada. Se trata, nada más y nada menos, que de un cogito, del cogito propiamente deleuziano que será pues denominado cogito para un yo disuelto. Partiendo de la hipótesis de lectura según la cual el capítulo 2 de Diferencia y repetición, "La repetición para sí misma", constituye una muy detallada exposición de dicho cogito, en las siguientes páginas nos proponemos explorar aquellos desarrollos, exhibiendo tanto la función que en él adquieren cada una de las facultades como sus relaciones y los efectos que producen las unas sobre las otras.

Palabras Clave: Deleuze, cogito, síntesis pasivas, síntesis temporales, teoría de las facultades

Abstract: Within the free and discordant relations that the faculties establish among each other, Deleuze outlines a particular experience, that of the genesis of thought, in which the faculties nevertheless maintain a fixed order: the origin of thought is to be found in the sensibility, which communicates its objet to the imagination, then to the memory, and finally to thought. And it is because of this invariance that such an

\footnotetext{
* Licenciado en filosofía por la Universidad de Buenos Aires y doctor en filosofía por la Universidad de Buenos Aires y la Université Paris VIII. Beneficiado de una beca doctoral del Consejo Nacional de Investigaciones Científicas y Técnicas de Argentina (CONICET), su tesis doctoral versó sobre la obra de Gilles Deleuze, y más precisamente, sobre la ontología diferencial que dicho autor desarrolla en Diferencia y repetición. Dirección electrónica: diego.abadi@gmail.com
} 
experience can be identified and named as that of a cogito, that of the Deleuzian cogito for a dissolved self. Following our hypothesis, according to which chapter 2 of Difference and repetition, "The repetition for itself", constitutes a very detailed exposition of such a cogito, in this paper we will try to explore those developments, exhibiting the emergence of the faculties of the Imagination, Memory and Thought, and the relations they establish with each other in the process.

Key words: Deleuze, cogito, passive synthesis, temporal synthesis, theory of the faculties

\section{Introducción: teoría de las facultades y cogito para un yo disuelto}

Sin lugar a dudas, el concepto más consistentemente criticado por Deleuze a lo largo de las numerosas páginas de Diferencia y repetición es el concepto de representación. Deleuze ataca a la representación desde diversos ángulos, según el problema enfocado, con el fin último de liberar a la diferencia y a la repetición del yugo al cual aquella las somete. En lo que respecta a lo que podríamos denominar como su aspecto subjetivo o mental, el pensamiento representativo ahoga a la diferencia y a la repetición bajo una doble identidad: la del sujeto pensante y la del objeto pensado. Y es justamente para oponerse a dicha concepción representativa del pensamiento que Deleuze desarrollará su propia teoría de las facultades. Ya no será cuestión de que las facultades trabajen en armonía para constituir un objeto idéntico bajo el modelo de la concordia facultatum, sino de que las facultades se relacionen violentamente de modo de establecer entre ellas un acuerdo discordante: cada facultad comunica a las demás su objeto particular, su dato propio, que, en tanto signo extraño, fuerza a la facultad que lo recibe a desplegar su ejercicio trascendente, yendo hacia su límite y buscando su objeto propio. Como resultado de ello, una nueva doctrina de las facultades tomará forma, definiéndose entonces cada facultad por su relación con un objeto que le concierna exclusivamente y que devendrá así imperceptible según su uso empírico. Deleuze enumera algunas de ellas: la sensibilidad y su objeto trascendente, el sentiendum como lo insensible; el pensamiento con su cogitandum como lo impensable; la imaginación con su imaginandum, el fantasma; la facultad del lenguaje, cuyo objeto, el loquendum, sería simultáneamente silencio; la vitalidad, cuyo objeto trascendente sería el 
monstruo; o la sociabilidad, que tendría como objeto a la anarquía o a la libertad.

Tres cosas pueden deducirse de esta nueva doctrina de las facultades: en primer lugar, que aquellas ya no se definen a partir de un sujeto del cual son los modos, sino que se encuentran liberadas de su anclaje subjetivo, fundándose solamente en la existencia de un objeto propio; en segundo lugar, que la lista de las facultades resulta así abierta, ya que la falta de un objeto trascendente correctamente definido podrá decretar la inexistencia de una facultad así como el descubrimiento de un objeto tal dará origen a una facultad nueva, anteriormente pasada por alto; en tercer lugar, que las facultades pueden relacionarse entre ellas de modo libre, ya que todas resultan heterogéneas las unas respecto de las otras. Ahora bien, a pesar de esta multiplicación y de esta liberación de facultades, en lo que respecta a una experiencia particular, la experiencia de la génesis del pensamiento, Deleuze mantendrá entre ellas una relación fija: el pensamiento tiene su origen en la sensibilidad, de allí se comunica a la imaginación, de ésta a la memoria y a continuación al pensamiento. Y esta tensión entre una libertad absoluta en la relación entre facultades y la ordenación fija que acabamos de presentar se justifica por lo siguiente: el movimiento de génesis del pensamiento constituye una experiencia privilegiada, que merece ser diferenciada. Se trata, nada más y nada menos, que de un cogito, del cogito propiamente deleuziano que será denominado cogito para un yo disuelto. En las siguientes páginas nos proponemos, entonces, explorar este cogito, exhibiendo tanto la función que en él adquieren cada una de las facultades como sus relaciones y los efectos que producen las unas sobre las otras. Antes de comenzar, sin embargo, resulta necesario llevar a cabo una larga lista de aclaraciones.

En lo que respecta al uso del término "cogito", cabe mencionar que esta generalización del término cartesiano corre por cuenta de Deleuze mismo. De hecho, comparándolo con el cartesiano, Deleuze se refiere a un cogito propiamente kantiano, así como cita a Ricœur y a su interpretación de la metapsicología freudiana como la exposición de un cogito abortado. En cuanto a la expresión "cogito para un yo disuelto", Deleuze la utiliza en dos ocasiones: en una para referirse al cogito propiamente kantiano, pero en la otra, en el prefacio, para referirse inequívocamente a su propio quehacer filosófico. ¿Qué sentido darle entonces a la noción genérica de "cogito"? Sin entrar en la especificidad del caso cartesiano, podríamos arriesgar la siguiente definición 
mínima: se trata de un movimiento que pretende llegar al extremo del pensamiento puro, de modo de acceder a una definición de derecho de lo que significa pensar que sirva para fundamentar sus usos empíricos. En el caso cartesiano, ese extremo lo provee el cogito ergo sum, que asegura la identidad del sujeto pensante, certeza apodíctica a partir de la cual se podrá fundar el edificio entero del conocimiento. En el caso deleuziano, en cambio, todo ello se ve invertido: ya no se tratará de arribar a un Yo idéntico, sino de conducir al pensamiento hasta el punto de su desfondamiento, de modo de acceder a un mundo a-subjetivo e impersonal en donde el Yo se disuelve.

Nuestra hipótesis de lectura será entonces que el capítulo 2 de Diferencia y repetición, "La repetición para sí misma", donde se exponen las tres síntesis del tiempo, constituye una muy detallada exposición de dicho cogito. A continuación, sin embargo, por una limitación espacial nos veremos obligados a exponer dicho capítulo sólo a medias. En las páginas que componen "La repetición para sí misma" Deleuze lleva a cabo una operación un tanto extraña: repite el mismo movimiento conceptual en dos niveles, uno que podríamos llamar filosófico y otro que habría que definir como psicoanalítico. Así, expone las tres síntesis temporales y su relación intrínseca una primera vez utilizando autores y recursos pertenecientes a la historia de la filosofía para a continuación repetir el mismo despliegue utilizando recursos psicoanalíticos. En las páginas que siguen nos ocuparemos solamente de la primera de ellas, la exposición filosófica. Dicha limitación inevitable cuenta por lo menos con un atenuante: en la medida en que la exposición psicoanalítica es expuesta en un segundo momento, de alguna manera la exposición filosófica le sirve de base. En ese sentido, el trayecto que presentaremos a continuación no estará en sí mismo incompleto, sino que nos brindará una primera versión que podrá ser luego complementada con los elementos psicoanalíticos restantes.

\section{La imaginación}

Deleuze es claro sobre el origen del pensamiento y, por tanto, sobre el punto de partida del cogito: "Es verdad que, en el camino que conduce a lo que queda por pensar, todo parte de la sensibilidad. De lo intensivo al pensamiento; siempre es por una intensidad que adviene el pensamiento". ${ }^{1}$ Pero si bien la

${ }^{1}$ Deleuze G., Diferencia y repetición, trad. María Silvia Delpy y Hugo Beccacece, Buenos Aires, Amorrortu, 2002, p. 223 [De aquí en más citado como DR]. 
sensibilidad es efectivamente el origen del cogito, ese lugar privilegiado la deja en una posición ambigua, ya que no es primera sino justamente porque no es todavía una facultad en sentido estricto. Habría que decir, entonces, que en el inicio del cogito la sensibilidad funciona como una proto-facultad, que no constituye su propio objeto, sino que simplemente lo recibe de modo pasivo. ${ }^{2}$ Será, por el contrario, gracias a la intervención de la imaginación que la sensibilidad orgánica tal como la conocemos tomará cuerpo en un complejo sensibilidad-imaginación. Veamos de dónde proviene esta "carencia" de la sensibilidad y de qué manera ella da lugar a las síntesis propias de la imaginación.

En el extracto citado anteriormente, Deleuze se refería a la intensidad como al objeto de la sensibilidad. Sin pretender definir la intensidad, concepto mayor de Diferencia y repetición que no podríamos abordar aquí, es necesario sin embargo subrayar una de sus características esenciales desde el punto de vista de la sensibilidad tal como la estamos abordando en este punto, a saber, su carácter instantáneo. La intensidad afecta pues a la sensibilidad bajo el modo de lo instantáneo: no recibimos una afección de la materia sin que la afección precedente no se haya ya desvanecido. De allí que la materia se conciba como mens momentanea, discontinua e incapaz de repetirse. En la medida en que cada nueva aparición implica la desaparición de la aparición anterior, la repetición se deshace mientras se hace, carece de en-sí, siendo cada instante independiente el uno del otro. Estando la materia entonces alienada, concebida como partes extra partes, la repetición no puede estar en los objetos, sino en el espíritu que los contempla y que extrae, de aquella discontinuidad, algo nuevo -una diferencia-, otorgándole el para-sí que originalmente no tenía. Ahora bien, ello no necesariamente implica postular que la repetición requiera, por parte de un sujeto activo, la subsunción del objeto repetido bajo un concepto idéntico, sino que implicará la contracción, por síntesis pasivas, de un sujeto contemplativo. ${ }^{3}$

${ }^{2}$ Dice Deleuze al respecto: "El privilegio de la sensibilidad como origen aparece en esto: que lo que fuerza a sentir y lo que sólo puede ser sentido son una sola y misma cosa en el encuentro, mientras que las dos instancias son distintas en otros casos." (DR, p. 223.)

${ }^{3}$ Deleuze definirá a la síntesis pasiva como una síntesis que no "está hecha por el espíritu, sino que se hace en el espíritu que la contempla, precediendo toda memoria y toda reflexión" (DR, p. 120). 
Es preciso preguntarse entonces cómo puede pasarse de esa discontinuidad de la materia en la sensibilidad, de esa repetición que no tiene en-sí, a la continuidad del objeto percibido, es decir, a su permanencia a través de las diferentes apariciones. Para dar respuesta a ese interrogante Deleuze se servirá de ciertos planteos humeanos y de las herramientas conceptuales que allí se desarrollan. Aunque tiene un objetivo diferente, a saber, el de cuestionar la noción de causalidad, y a partir de ello el estatuto general del conocimiento, la pregunta inicial de Hume es la misma que la pregunta deleuziana. El primero se pregunta cómo a partir de impresiones que son, de derecho, independientes, puede extraerse una impresión cualitativa en la cual ambas estén unidas, siendo que de esa impresión cualitativa podrá deducirse una relación de causalidad. Pero si bien así expresado parece un problema abstracto, lo que se propone Hume es apuntar, por el contrario, a los casos más cotidianos de nuestra experiencia. Así por ejemplo, si tengo un vaso con agua y lo inclino (impresión A), el agua cae al suelo (impresión B), y el hecho de haber inclinado un vaso con agua un número suficiente de veces me conduce a pensar que cada vez que haga lo primero, sucederá lo segundo. Si A y B son impresiones independientes, ya que puedo representarme y experimentar a la una sin la otra, Hume se pregunta entonces de dónde viene mi certeza de que, cada vez que incline un vaso con agua su contenido caerá al piso. Y como consecuencia de esa primera cuestión, se pregunta también de qué tipo de certeza se trata en dichos casos, ya que no se trata de una certeza fruto de la reflexión, sino que es, en cierto sentido, previa a dicha operación del entendimiento: vemos que un niño está por inclinar su vaso, y antes y sin necesidad de reflexionar sobre sus posibles consecuencias, reaccionamos para evitarlo ya que "sabemos", inmediatamente, qué sucederá tras aquella acción.

Hume responde que es la imaginación, mediante la contracción, la responsable de aquella fusión de impresiones distintas en un solo caso, y que la certeza que experimentamos al percibir alguna de esas impresiones viene dada por el hábito. Así, ante la recurrencia de dos apariciones contiguas AB, el complejo $\mathrm{AB}$ se funde en la imaginación que, como una "placa sensible", retiene el uno cuando el otro aparece, generando el hábito de esperar la aparición de $\mathrm{B}$ cuando $\mathrm{A}$ aparece. La contracción retiene los elementos particulares ya repetidos, dando lugar a una dimensión del pasado, el pasado de la retención, mientras que la diferencia sonsacada a los casos particulares da como resultado la dimensión del futuro inmediato, bajo la forma de la espera. 
Pero, según Hume, para que la síntesis de la imaginación logre extraer de los casos contraídos una verdadera generalidad, es decir, la aserción general que afirme que "cada vez que aparezca $A$, también aparecerá B" $(A \rightarrow B)$, es necesario que las síntesis propias de la memoria y del entendimiento se funden sobre la primera y la prolonguen. En dicho proceso, la memoria distingue los elementos (A y B) que en la imaginación se encuentran fundidos en una sola impresión cualitativa $(\mathrm{AB})$, transformando el pasado inmediato de la retención en un pasado de la reflexión en el que cada elemento particular puede ser reproducido; el entendimiento, por su parte, eleva el futuro inmediato de la anticipación hasta un futuro de la previsión, que permite pasar de la espera a la generalización.

Para Deleuze, sin embargo, aquí Hume avanza demasiado rápido, ya que si bien capta muy bien la especificidad de la imaginación, da de ella sin embargo una versión limitada: por un lado, acota su esfera de influencia al campo de la percepción, sin retroceder lo suficiente como para llegar hasta la fundación misma de la contracción; por otro, al prolongar las síntesis de la imaginación en las síntesis de la memoria y del entendimiento, mezcla síntesis pasivas y síntesis activas sin tener en cuenta su diferencia de naturaleza. Corrigiendo esa primera limitación, y extendiendo la contracción de la imaginación hasta sus bases, Deleuze descubrirá entonces el terreno de las síntesis pasivas, y, particularmente, el del primer tipo de ellas. Así, si se considera que Hume presenta una repetición de casos, siendo la diferencia que se extrae de ellos una generalidad, a través del aporte de Bergson la contracción de la imaginación puede extenderse hasta un nivel anterior, la de la repetición de elementos. Bergson propone el siguiente ejemplo: son las cuatro de la tarde, y el reloj lo indica mediante cuatro campanadas. Ellas son independientes las unas de las otras, mens momentanea (a, a, a, a), pero nosotros las contraemos en una "impresión cualitativa interna" que hace de ellas cuatro campanadas $(\mathrm{a}+\mathrm{a}+\mathrm{a}$ $+\mathrm{a}=4 \mathrm{a}$ ). Mediante un ejemplo un tanto forzado, los planteos de Hume y de Bergson pueden mostrar su complementariedad: tras dar las cuatro de la tarde mediante las campanadas, y sólo en ese momento del día, el reloj hace cucú. Así pues, si las cuatro campanadas conforman el elemento "cuatro de la tarde" (A), el hecho de que a las cuatro de la tarde el reloj haga cucú conforma el caso general "a las cuatro de la tarde el reloj hace cucú" (AB). La contracción de los casos, entonces, supone siempre una contracción de elementos, mientras que 
la contracción de elementos puede a su vez continuarse en todo tipo de contracciones de casos.

Ahora bien, esta distinción relativa entre casos y elementos permite vislumbrar, por regresión, una contracción elemental. Y es el modelo de esa contracción en su nivel más elemental el que nos permitirá obtener una caracterización de la primera síntesis pasiva. Se trata, en primer lugar, de una síntesis constituyente. Así, ante la pregunta, ¿cómo funciona la síntesis?, el modelo de la contracción ya nos proveía una respuesta: la contracción es la integración, en una impresión cualitativamente distinta, de elementos heterogéneos o autónomos. Pero en la medida en que la síntesis pasiva es constituyente, cabe preguntarse cómo se constituye ella misma. Para responder a ello, hay que subrayar y sostener la ambivalencia del genitivo al hablar de una contracción de elementos. Es decir, si se quiere pensar una contracción elemental y constituyente, hay que pensar no sólo una contracción primera que enfoque posteriormente ciertos elementos, sino también a esos elementos contrayéndose en sí mismos. Así pues, como condición de la sensibilidad o la percepción empíricas, hay que postular una contracción material fundamental, la de "una sensibilidad primaria que somos" una vez que la imaginación logra fundirse con la sensibilidad: "Somos agua, tierra, luz y aire contraídos, no sólo antes de reconocerlos o representarlos, sino antes de sentirlos. Todo organismo es, en sus elementos receptivos y perceptivos, pero también en sus vísceras, una suma de contracciones, retenciones y esperas". ${ }^{4}$

Esta contracción elemental produce una subjetividad particular, la del hábito como fruto de la contemplación, y da forma a un sujeto, el yo pasivo, que hay que diferenciar del sujeto tal como se lo concibe tradicionalmente. Si este último, en tanto Yo activo o agente de síntesis, se caracteriza por su espontaneidad y tiene como su operación propia a la reflexión formal, separable de todo contenido o materia, la contemplación que define al yo pasivo tiene en su origen siempre una materia, aunque heterogénea, a partir de la cual extrae una imagen de sí mismo: "No nos contemplamos a nosotros mismos, pero no existimos más que contemplando, es decir, contrayendo aquello de lo cual procedemos, (...) es siempre otra cosa, es el agua, Diana o los bosques lo que debe contemplarse antes que nada, para llenarse de una imagen de sí mismo". ${ }^{5}$

\footnotetext{
${ }^{4}$ DR, p. 123.

${ }^{5}$ DR, p. 125.
} 
Es entonces la operación de la contemplación la que da lugar al hábito que designará un yo pasivo. O para decirlo de modo más sencillo: un yo pasivo, un sujeto larvario, es un hábito, el hábito de contraer una determinada materia de una determinada manera. Así, entre la contracción de la contemplación y la contracción del hábito puede reconocerse una diferencia de potencia, constituyendo el hábito una contracción de segundo grado con respecto a la contracción primaria de la contemplación. $\mathrm{O}$, retomando los términos de Hume y Bergson, el hábito contrae los casos mientras que la contemplación contrae los elementos. Se trata, sin embargo, tal como lo hemos visto, de una diferencia relativa, una diferencia de grado y no de naturaleza, ya que un caso puede ser el elemento de un caso más general, y viceversa. "Cuando decimos que el hábito es contracción -dice Deleuze-, no hablamos, por consiguiente, de la acción instantánea que se compone con la otra para formar un elemento de repetición, sino de la fusión de esta repetición en el espíritu que contempla. Es preciso atribuir un alma al corazón, a los músculos, a los nervios, a las células, pero un alma contemplativa cuyo rol se limita a contraer el hábito". ${ }^{6}$ Así, el hábito del corazón vendrá dado por una contracción particular, la de contraerse y distenderse de modo de bombear la sangre para irrigar el resto del cuerpo, o el hábito de los músculos vendrá dado por la contracción o distención de sus tejidos con el objetivo de generar el movimiento del cuerpo. Pero tal como puede deducirse de los ejemplos, el hábito se monta sobre una primera contracción, que les provee sus objetos propios, y estos objetos, constituidos por la síntesis contemplativa, se caracterizarán como signos, ya que el signo se define, para Deleuze, como el efecto de "una comunicación que se establece entre elementos dispares". 7

Y en la medida en que esta síntesis pasiva es constituyente, y previa a la actividad del espíritu, ella constituye una síntesis temporal, la síntesis del presente viviente. La contracción, fundiendo los instantes entre sí, constituye el tiempo presente, del cual el pasado y el futuro son sus dimensiones: el pasado como la retención de los instantes particulares en la contracción y el futuro como la anticipación orientada a lo general. Si volvemos al ejemplo de Bergson, la contracción de las cuatro campanadas provee de cierto espesor al presente: las campanadas pasadas, aun pasadas, siguen formando parte de la impresión

\footnotetext{
${ }^{6} \mathrm{DR}, \mathrm{p} .124$.

${ }^{7}$ DR, p. 48.
} 
presente de las cuatro campanadas, así como lo hacen las campanadas que resten y que son esperadas al aparecer la primera de ellas. Pero tal como lo consigna el hecho de que se trate de un presente viviente, la contracción del presente tiene como condición que haya una individuación viviente, actual, que pueda contemplar la contracción. Así, lo que para un individuo puede ser todavía presente, para otro individuo de distinta escala puede ya ser pasado, y viceversa. Y si se pregunta, por tanto, si puede haber un perpetuo presente, lo que implicaría concebir una contemplación que se extienda al infinito de los instantes, habrá que responder que ello es contradictorio, ya que la contracción opera siempre sobre elementos o casos dados.

A partir de lo anterior, la receptividad deviene entonces un campo complejo, compuesto de diferentes niveles de contracciones. Por una parte, entre los distintos niveles se conforma un campo de signos naturales, que envuelven tanto los elementos heterogéneos de un mismo nivel como los niveles heterogéneos entre sí. Estos se definen como naturales porque pertenecen siempre al tiempo presente, mientras los signos artificiales, fruto de las síntesis activas, remiten tanto al pasado como al futuro. Por otra parte, allí donde hay una contemplación hay un hábito, y por tanto, para Deleuze, un yo pasivo o un sujeto larvario. En su guía de lectura de Diferencia y repetición, James Williams resume en los siguientes puntos algunas de las conclusiones más relevantes que pueden extraerse de la caracterización de esta primera síntesis: 1) entre los diferentes niveles de síntesis pasivas no hay una relación de causalidad, sino de condicionamiento, ya que cada nivel tiene un contenido radicalmente diferente al del resto; 2) los signos no son objetos que puedan ser representados y conceptualizados, de hecho, el signo se desvanece cuando intentamos conocerlo. Tiene que haber, por tanto, un acercamiento distinto a los signos, hay que dejar que estos "nos trabajen" ("work through us"), ya que los hábitos no surgen de la actividad sino de la contemplación; 3) los sujetos larvarios o los yoes pasivos aparecen como bien definidos en un solo nivel de síntesis, tornándose confusos desde la perspectiva de los otros niveles que implica o en los cuales se encuentra implicado. ${ }^{8}$

Vemos pues que esta primera síntesis, comandada por la imaginación pero dando como resultado una integración entre la imaginación y la

8 Williams J., Gilles Deleure's Difference and repetition: a critical introduction and guide, Edinburgh, Edinburgh University Press, 2003, p. 91. 
sensibilidad, compone un campo receptivo complejo y heterogéneo. A diferencia, por ejemplo, de la receptividad kantiana, no se contenta con recibir datos ya informados espacio-temporalmente sino que produce gradualmente un campo espacio-temporal complejo, constituido por diversos niveles de síntesis, cada uno de los cuales resulta dependiente de alguna materia determinada. Así pues, ya en el primer momento del cogito deleuziano la soberanía del Yo idéntico se ve socavada, ya que si no nos dejamos engañar por las síntesis activas del entendimiento, que se representan como primeras pero que, en el orden de la génesis, resultan derivadas, nos encontramos en el origen del cogito con una multiplicidad de sujetos larvarios, de almas contemplativas pasivas, que disuelven al Yo en un universo material y prepersonal.

\section{La memoria}

Pero, tal como habíamos adelantado, en el cogito la comunicación entre las facultades responde a algún tipo de necesidad. En este caso, lo que fuerza a entrar en el terreno de la memoria es una limitación inherente a las síntesis de la imaginación. Tal como hemos visto, el presente viviente resulta necesariamente finito, ya que la contracción tiene un límite en la fatiga, a la vez que está condicionada por los elementos heterogéneos a contraer. Es decir, si bien aquella síntesis constituye cada vez el presente, todo presente constituido pasa, y ese pasar remite a una segunda síntesis que funciona como el fundamento de la primera: "El hábito -dice Deleuze- es la fundación del tiempo, el suelo móvil ocupado por el presente que pasa. (...) Pero lo que hace pasar el presente, y lo que apropia el presente y el hábito, debe estar determinado como fundamento del tiempo. El fundamento del tiempo es la Memoria". Así pues, la primera síntesis debe ser caracterizada como intratemporal, en la medida en que se constituye sobre presentes que pasan. Pero afirmar que "la Memoria es la síntesis fundamental del tiempo, que constituye el ser del pasado", ${ }^{10}$ abre a su vez nuevas preguntas: ¿cómo caracterizar el ser del pasado?, ¿a qué tipo de memoria se refiere Deleuze?

\footnotetext{
${ }^{9}$ DR, p. 133.

${ }^{10}$ DR, p. 133.
} 
Según la lógica que guiaba la exposición de la primera síntesis, la Memoria buscada no puede confundirse con la memoria activa, sino que deberá referirse a una Memoria constituyente y pasiva. En este caso, sin embargo, expondremos primero la memoria activa ya que, una vez identificado su funcionamiento, la búsqueda de sus condiciones nos conducirá a la caracterización de la Memoria trascendental. Sin embargo, a diferencia de la primera síntesis, ambas tienen un carácter común, que permite reconocer un punto de partida compartido. Dentro de la perspectiva del presente viviente, pasado y futuro no eran más que dimensiones en las cuales se extendía el presente, siendo el pasado lo particular retenido en la contracción, así como el futuro era lo general abierto por la espera. Según la segunda síntesis temporal, en cambio, es el pasado lo que se transforma en el elemento de lo general, mientras que el presente se vuelve particular. Desde la perspectiva de las síntesis activas, el pasado, en tanto elemento, está entre dos presentes: entre el presente que ya pasó -el antiguo presente-, y el presente actual con respecto al cual el anterior ha pasado. Pero el pasado no se identifica al antiguo presente, sino que es el elemento, es decir, el medio en el cual aquel se encuentra emplazado y gracias al cual puede ser enfocado. Así, el antiguo presente, en tanto lo enfocado, será lo particular, mientras que el elemento en sí será lo general. Es entonces gracias al elemento del pasado, que conserva todos los antiguos presentes particulares, que cualquiera de ellos puede ser enfocado y reproducido por la memoria activa.

Pero esta reproducción, que define la actividad de la memoria, requiere de otra actividad que le es complementaria, la de la reflexión, a cargo del entendimiento. Y es en la conjunción de estas dos actividades que se obtiene la operación de la representación. Cuando se reproduce un antiguo presente, se lo enfoca en el elemento del pasado, de manera tal que aquel presente se encuentra representado en el presente actual desde el cual es enfocado. Los límites de esa reproducción dependen de las semejanzas o contigüidades que puedan mantenerse entre la representación actual y el antiguo presente representado, factores que definen las relaciones psicológicas de asociación. Ahora bien, para que pueda producirse la reproducción es necesario que también tenga lugar la reflexión, ya que no sucede que el antiguo presente esté representado en el actual sin que, a su vez, el actual no esté representado en esa misma representación. Tal como lo afirma Deleuze: "Es misión esencial de la representación representar no solamente algo, sino su propia 
representatividad". ${ }^{11}$ Así pues, el presente antiguo y el actual no se ordenan como instantes sucesivos en una línea de tiempo, sino que el actual "contiene necesariamente una dimensión más por la cual re-presenta el antiguo, y en el cual se representa a sí mismo". ${ }^{12}$ Volviendo entonces sobre aquella primera síntesis, puede ahora hacerse un contraste entre las síntesis pasivas y las síntesis activas. A partir de la actividad del reconocimiento, la síntesis activa retoma el material de las síntesis pasivas de la imaginación y se traslada del pasado inmediato en la retención al pasado reflexivo de la representación. El futuro deja de ser el futuro inmediato de la anticipación para ser el futuro reflexivo de la previsión. Pero si esta re-presentación de sí mismo del presente actual, es decir, la reflexión, es condición de la reproducción de la memoria activa, cabe preguntarse si la reflexión no tiene, a su vez, ella misma una condición. Deleuze responderá que sí, y que esa condición es la "existencia" del pasado en sí, ya que es ésta la que provee el paso que la reflexión supone: "Tal presente antiguo resulta ser reproducible y el actual presente puede reflejarse gracias al elemento puro del pasado, como pasado en general, como pasado a priori". ${ }^{13}$ Una vez más, entonces, las síntesis activas desfiguran un orden de prioridad genético. La reflexión no funda la memoria a través de su propia representación, sino que hay otro tipo de memoria que provee un paso que es condición de la propia representación. Así, la identidad en la reflexión supone la temporalidad.

Para caracterizar este pasado puro o a priori, Deleuze se sirve de tres paradojas que encuentra en Bergson: la de la contemporaneidad, la de la coexistencia y la de la preexistencia. Y así las denomina Deleuze ya que lo afirmado por ellas resultará paradójico desde el punto de vista de la representación, que no puede representarse el pasado más que como un antiguo presente, a la vez que tiene que suponer un elemento puro del pasado que no se deja concebir de aquella manera. La paradoja de la contemporaneidad, en primer lugar, afirma que el pasado es contemporáneo del presente, es decir, que no se constituye después del presente. Hay que afirmar esta contemporaneidad ya que, si para que existiera el pasado hiciera falta que, en un primer momento, un presente pasara, aquel paso presupondría lo que se pretende probar, ya que, para que el presente se trasforme en pasado,

\footnotetext{
${ }^{11}$ DR, p. 134.

${ }^{12}$ DR, p. 134.

${ }^{13}$ DR, p. 135.
} 
aquel debería ser reemplazado por un nuevo presente. Pero para que pueda aparecer un nuevo presente, el presente anterior ya tiene que haber pasado, porque de lo contrario no habría lugar para la aparición del nuevo presente. Por lo tanto, no se puede partir del presente para constituir el pasado, sino que hay que suponer que el pasado es contemporáneo del presente. La paradoja de la coexistencia afirma, por su parte, que el pasado se conserva en sí mismo, de manera que todo el pasado coexiste consigo mismo. Lo que, a su vez, permite modificar el sentido de la contracción, en comparación con el modo en el que esta se comprendía en la primera síntesis. Si el pasado es una totalidad que conserva todos los antiguos presentes, y el presente actual es un particular, habrá que postular que el presente actual es entonces el grado más contraído de ese pasado puro. Ahora bien, ello no significa borrar la diferencia de naturaleza entre ellos: el pasado en sí es un pasado que nunca fue presente, insistiendo o consistiendo con los presentes que pasan. La última paradoja se revela entonces como una consecuencia de las dos anteriores: el elemento puro del pasado preexiste al presente que pasa. Así pues, hay "un elemento sustancial del tiempo (pasado que no fue nunca presente) que desempeña el papel de fundamento, y que no está, él mismo, representado". ${ }^{14}$ La representación, por su parte, sólo se representa presentes, actuales o antiguos, mientras que presupone este elemento del pasado puro que no puede sin embargo ser representado.

Las consecuencias que pueden extraerse de esto son considerables. Si todo el pasado coexiste con el presente, que se concibe como su grado más contraído, el pasado conserva en sí todos los grados de contracción y distensión posibles. De tal manera que cada presente puede considerarse como contrayendo distintos niveles del pasado. Más aún, habría que decir que cada presente contrae necesariamente distintos niveles de pasado, ya que cada uno de ellos es, en sí mismo, una contracción relativa, que recupera e implica niveles más distendidos, mientras que es implicada y recuperada por los niveles más contraídos, aquellos que se encuentran más cercanos al presente actual. Así, si se analiza lo anterior desde la perspectiva de una vida, pueden reconocerse dos caracteres distintos de la contracción, uno empírico y otro nouménico. El carácter empírico define la contracción que tiene lugar entre los instantes que se suceden, entre las relaciones de causalidad y contigüidad que los vinculan,

${ }^{14}$ DR, p. 136. 
pero esa sucesión de presentes conduce a su vez a plantear otro tipo de vínculo, el nouménico, del cual la contracción empírica sería su manifestación. Sumando este segundo punto de vista puede decirse que una vida tiene un destino, es decir, que los hechos que desde la perspectiva empírica aparecen aislados entre sí guardan sin embargo relaciones más complejas, de resonancia o de acción a distancia, de modo tal de expresar la continuidad de un mismo destino. Así, inadvertidamente, si el pasado en sí es la condición de la representación, operación esencial para asegurar la identidad del sujeto, este elemento puro hunde al sujeto en un fondo espiritual en el cual aquella identidad se desvanece. Ya que, tal como lo aclara Deleuze, "lo que decimos de una vida podemos decirlo de varias vidas. Puesto que cada una es un presente que pasa, una vida puede retomar otra, en otro nivel: como si el filósofo y el cerdo, el criminal y el santo desempeñasen el mismo pasado, en los diferentes niveles de un cono gigantesco. Es lo que se llama metempsicosis". 15

Definido entonces este elemento del pasado en sí, cabe preguntarse, por una parte, cuál es el objeto trascendente de la Memoria, y por otra, cómo puede ésta última experimentar aquel elemento sub-representativo del pasado en sí. Y si Bergson le servía a Deleuze para definir este elemento, será Proust quien proveerá las herramientas para responder a estas preguntas. En lo que respecta a la segunda de ellas, siguiendo a Proust, Deleuze retomará la noción de memoria involuntaria. Esta, en lugar de estar sometida a los dictados de la representación, que la manda a buscar recuerdos particulares, antiguos presentes que por alguna relación de semejanza o utilidad son requeridos por el presente actual, presenta, involuntariamente, bloques de pasado en sí, es decir, de recuerdos que nunca fueron presentes. En el caso de Proust, el recuerdo involuntariamente surgido por la magdalena no es el de Combray tal como efectivamente fue experimentado, sino el de un Combray en sí, "en un esplendor nunca vivido". ${ }^{16}$ Pero en lo que respecta a la primera pregunta planteada, aquella por el objeto trascendente de la Memoria, Proust también provee indicaciones preciosas, al mostrarnos que aquella reminiscencia es siempre erótica: Eros es el noúmeno, lo buscado en ese pasado en sí, mientras que Mnemosine es la encargada de sumergirse en ese elemento puro,

\footnotetext{
${ }^{15}$ DR, p. 138.

${ }^{16}$ DR, p. 140.
} 
explorándolo bajo la forma de la repetición. Así pues, el objeto de la memoria trascendental es lo inmemorial. Y así como mencionamos que una vida se abría a infinitas vidas en la metempsicosis, el objeto amado buscado por Mnemosyne no es tampoco un objeto individualizable ni tiene un origen determinado, sino que es siempre ya una repetición que nos devuelve a un campo intersubjetivo infinito:

no hay término último, nuestros amores no remiten a la madre; simplemente, la madre ocupa en la serie constitutiva de nuestro presente un lugar con respecto al objeto virtual, que es necesariamente ocupado por otro personaje en la serie que constituye el presente de otra subjetividad, considerando los desplazamientos de ese objeto $=\mathrm{x}$. Un poco como el héroe de la Búsqueda que, al querer a su madre, repite ya el amor de Swann por Odette. ${ }^{17}$

En esta segunda escala del cogito, comandada por la Memoria, pasamos pues de lo material a lo espiritual. Si en el caso del Habitus y la imaginación la identidad del Yo se veía fragmentada en los múltiples sujetos larvarios que acercaban el sujeto a la materia, en el caso de la Memoria la identidad se disuelve en la totalidad espiritual, de la cual un sujeto individual no conforma más que la contracción máxima de algún nivel determinado. Queda por ver, entonces, de qué manera la Memoria y el pasado requieren a su vez de una tercera síntesis, que evite la totalización que una hipóstasis de la Memoria amenaza con establecer.

\section{El pensamiento puro}

Detener el recorrido en la Memoria dejaría a Deleuze en un punto del cual él justamente desea escapar: el de una totalidad omniabarcativa que, en tanto repositorio nouménico, cumpliría el rol de fundamento con respecto a todos los fenómenos nuevos que vinieran a la existencia. Para evitar esta deriva, la facultad del pensamiento tiene que lograr perforar la totalidad espiritual del pasado en sí, operación que logrará llevar a cabo gracias al descubrimiento de una nueva síntesis temporal que se mantiene latente bajo las dos síntesis anteriores. Y si bien según Deleuze las facultades nunca están dadas de

${ }^{17}$ DR, p. 167. 
antemano, sino que tienen que constituirse a través del contacto con su objeto trascendente, en el caso del pensamiento esta aserción toma una fuerza mayor. En su crítica a los postulados que conforman la imagen del pensamiento, Deleuze insiste en que el pensamiento no es algo naturalmente dado, sino que por el contrario hay que hacer nacer al pensar en el pensamiento, y la experiencia que dará origen al pensar será, justamente, el encuentro con un tiempo particular que no es el tiempo ni del Hábito ni de la Memoria.

Para acceder a este tipo particular de tiempo será útil trazar una comparación con el tiempo que puede hallarse en la reminiscencia platónica. Ésta efectivamente incluye al tiempo en el pensamiento, ya que si pensar es recordar, ello implica introducir un tiempo anterior, olvidado, hacia el cual el pensamiento debe remontarse. Pero lo que hay que preguntarse es, sin embargo, de qué manera la reminiscencia introduce el tiempo. O lo que es lo mismo, ¿de qué tipo de tiempo se trata en ese caso? Para Deleuze, estamos ante "un tiempo físico, un tiempo de la Physis, periódico o circular, subordinado a los acontecimientos que transcurren en él o a los movimientos que mide". ${ }^{18}$ Es decir, un tiempo aristotélico, número del movimiento, que no deja de ser segundo con respecto a los contenidos que se desarrollan en él.

Pero hay, sin embargo, otro concepto de tiempo, de un tiempo puro, no subordinado a lo que sucede en él, que estará asociado no ya a la Memoria sino al pensamiento puro y cuyo descubrimiento Deleuze ubica en la obra de Kant. Este tiempo puro emerge justamente en la comparación del cogito de éste último con el cogito cartesiano. El cogito cartesiano tenía dos valores: la existencia indeterminada que hay que determinar, el "yo soy", y la determinación dispuesta para llevarlo a cabo, el "yo pienso". La determinación implica una existencia determinada, ya que "para pensar hay que ser", a la vez que determina a esa existencia directamente, ya que del "pienso, luego soy", Descartes deduce el "soy una cosa que piensa". La crítica de Kant apunta justamente a ese modo de determinación según el cual la determinación ("pienso") se predica directamente de lo indeterminado ("soy una cosa que piensa"). Para Kant en el cogito cartesiano falta explicitar un tercer valor, el de lo determinable, es decir, el de la forma bajo la cual lo indeterminado es determinable por la determinación. Y la forma bajo la cual lo indeterminado será determinable es justamente la forma del tiempo. En términos kantianos,

${ }^{18}$ DR, p. 145. 
ello conduce a la paradoja del sentido interno. Paradoja que afirma que "mi existencia indeterminada no puede ser determinada más que en el tiempo, como la existencia de un fenómeno, de un sujeto fenomenal, pasivo o receptivo que aparece en el tiempo". ${ }^{19}$ Así, la espontaneidad de la cual me apercibo en el "Yo pienso" no puede postularse como el atributo de un ser sustancial, sino que debe concebirse como "la afección de un yo [mol] pasivo que siente que su propio pensamiento (...), aquello por lo cual dice YO [Je], se ejerce en él y sobre él, y no por él". ${ }^{20}$ En este punto pues, la distinción entre el Yo [Je] y el yo [moi] - para la cual el español no dispone lamentablemente de términos distintos- se hace esencial. Según esta versión kantiana del cogito, el yo que piensa no es idéntico a sí mismo. Por el contrario, el yo que piensa está esencialmente fisurado: la actividad espontánea del pensamiento, esto es, la posibilidad de decir "Yo pienso", requiere de una afección previa, pero que afecta al yo pasivo; por otra parte, el "Yo pienso" se aplica a ese yo pasivo, pero que, desde su pasividad, la experimenta como llevada a cabo por una entidad activa y que, por lo tanto, le es heterogénea. Así pues, entre la existencia indeterminada del "soy", y la determinación del "pienso", hay que agregar el yo [moi], como instancia intermedia. Y el modo mediante el cual el yo pasivo puede ser determinado por el Yo activo es justamente el tiempo. En contraposición a lo que sucedía en Descartes, en cuyo caso el "pienso" afectaba al yo instantáneamente, para Kant, entre el yo pasivo y el Yo activo hay una diferencia temporal. En el cogito cartesiano, el Yo conservaba su identidad gracias al carácter instantáneo de su determinación, mientras el tiempo quedaba en manos de Dios, que funcionaba como el garante de aquella identidad gracias a una creación continua mediante la cual todos los instantes en los que se podía decir "yo pienso" formaban una continuidad. Kant, por el contrario, al incluir el valor de lo determinable, es decir, del tiempo, fractura la identidad del Yo, anticipándose, implícitamente, a la famosa frase de Rimbaud: Yo es otro.

Es por esto que Deleuze considera que "la mayor iniciativa de la filosofía trascendental consiste en introducir la forma del tiempo en el pensamiento como tal", 21 y la razón por la cual, a pesar de sus múltiples críticas a Kant, mantiene la noción de "trascendental" al momento de referirse a su propia

\footnotetext{
${ }^{19}$ DR, p. 142.

${ }^{20}$ DR, p. 142.

${ }^{21}$ DR, p. 143.
} 
obra. Y si la introducción del tiempo en el pensamiento como tal es atribuida a Kant y no, por ejemplo, a Platón, ello se debe a que el tiempo que introduce la filosofía trascendental es muy distinto al tiempo circular que se desprendía de la segunda síntesis. Este tiempo, al que nos conduce a la fisura del Yo, es la forma vacía del tiempo. Es decir, se trata de un tiempo que ya no es más la medida del movimiento o la forma de un contenido físico que la precede, sino que es un tiempo puro, línea recta previa a y condición de la physis. ${ }^{22}$ Esa es pues la forma primera del tiempo, y es la que, por una parte, fisura al Yo exponiéndolo a lo que Hölderlin denominará desvío categórico-, ${ }^{23}$ dejando tras su paso dos instancias heterogéneas (Yo/yo), dos mitades de un símbolo que no encastran la una en la otra, y la que, a la vez, en tanto forma de lo determinable, deviene condición de la determinación de una por la otra.

Así pues, con la introducción de la forma vacía del tiempo llegamos, tras un recorrido por momentos sinuoso, a un punto de inflexión en nuestro camino. El Hábito nos proveía una diferencia sonsacada y una repetición desnuda, que requerían como su condición a la Memoria, con su diferencia como variante y su repetición vestida. Pero esta última, tal como vimos, tendía a convertirse en un fundamento, tornando a la diferencia y a la repetición relativas a su contenido que, interiorizado, formaba un Todo. Es entonces solamente con el descubrimiento de la forma pura del tiempo que se accede por primera vez a la Diferencia en sí. Ella "constituye el descubrimiento de la Diferencia, no ya como diferencia empírica entre dos determinaciones, sino Diferencia trascendental entre LA determinación y lo que ella determina, no ya como diferencia exterior que separa, sino Diferencia interna, y que relaciona $a$ priori el ser y el pensamiento el uno con el otro". ${ }^{24}$ Estamos pues ante un tipo de síntesis que se distingue considerablemente de los dos tipos de síntesis anteriores. Se trata de una síntesis particular ya que sintetiza sin unificar, y sin ninguna condición de semejanza previa, sino a través de una diferencia interna que relaciona lo diferenciado.

Con la aparición de esta nueva forma temporal se opera pues una reorganización de las distintas síntesis temporales en sus relaciones mutuas. Para referirse a esto último, Deleuze recurre a tres modos de definir el tiempo,

${ }^{22}$ DR, p. 145.

${ }^{23}$ Beaufret J., Hölderlin et Sophocle, Brionne, G. Monfort, 1983.

${ }^{24}$ DR, p. 141. 
el orden, el conjunto y la serie. Aquí, sin embargo, sólo nos detendremos en el último de ellos, en la medida en que subsume a los anteriores. Recogiendo al orden y al conjunto del tiempo, la serie del tiempo redistribuye la desemejanza del antes y el después, reabsorbiendo las dos síntesis temporales precedentes y encajando sus distintos tipos de repeticiones. Según la serie del tiempo, lo que define a priori el pasado o lo anterior es la repetición por defecto. El pasado es, pues, el tiempo en el cual la acción formidable se concibe como "demasiado grande para mí". Sin embargo, esta repetición por defecto es la que prepara para la acción: es la condición bajo la cual algo nuevo puede producirse. Así, si se toman ciertos casos históricos como ejemplo de lo anterior, entre Lutero y Pablo o entre la revolución del 89 y la república romana no debe postularse una relación de semejanza, fruto de la reflexión de los historiadores y sus analogías extrínsecas. Hay que pensar, por el contrario, que Lutero y los revolucionarios del 89 se viven a sí mismos como repitiendo a Pablo y a la república romana, antes de, y como condición para, ser capaces de llevar adelante sus acciones revolucionarias propias. Lo que define, por su parte, al tiempo de la cesura o la fisura, es el "devenir-igual a la acción", 25 el desdoblamiento del yo gracias al cual nos transformamos en capaces de realizar la acción formidable. Por último, el tercer tiempo, síntesis del porvenir, nos abre a un tiempo en el cual "el acontecimiento, la acción, tienen una coherencia secreta que excluye la del yo, que se vuelve contra el yo convertido en su igual y lo proyectan en mil pedazos (...): el yo se ha igualado con lo desigual en sí”. ${ }^{26}$ Se trata, pues, en este caso, de una repetición por exceso, que se libera de sus condiciones y de sus agentes.

Es entonces el pensamiento puro el que puede hacer esta experiencia extrema de despersonalización, afrontando la disolución del Yo [Je] y el yo [moi] y asomándose así al mundo de las singularidades preindividuales y de las individuaciones impersonales. De hecho, hacer nacer al pensamiento implica justamente dotar al pensamiento de la capacidad de enfrentarse a este tercer tiempo, de modo de lograr "abstraer el tiempo de todo contenido para desprender de él la forma pura". ${ }^{27}$ Pero en la medida en que el pensamiento puro es una facultad, debe dar consistencia a ese contacto con su propio tipo

\footnotetext{
${ }^{25}$ DR, p. 146.

${ }^{26}$ DR, p. 147.

${ }^{27}$ DR, p. 179.
} 
de diferencia a través de una repetición característica que logre determinar aquel tercer tiempo. ¿Cómo comprender, entonces, su tipo propio de repetición, el de la repetición por exceso? Si la primera síntesis constituía una repetición física, y la segunda una repetición espiritual o metafísica, la tercera síntesis constituye, por su parte, una repetición ontológica. Es decir, nos da la repetición primera, la que definirá al Ser en tanto tal. Y esta repetición ontológica, que conjuga, paradójicamente, la máxima potencia de destrucción con la más ilimitada potencia de producción de lo nuevo, Deleuze la encuentra anticipada por la noción nietzscheana de Eterno Retorno. Si bien según su propia presentación, Deleuze se propone retomar y explicitar aquella noción que en Nietzsche había quedado en un estado implícito o larvario, ${ }^{28}$ por nuestra parte encontramos más útil marcar, al menos para una exposición sintética de ella, su punto de divergencia más que su relación de continuidad. Así, creemos que la versión propiamente deleuziana del eterno retorno se esclarece si se pone de relieve una diferencia esencial, aun si ésta fuera meramente nominal: si Nietzsche se refirió a la noción en cuestión como Eterno Retorno de lo Idéntico, la versión deleuziana podría bien caracterizarse, invirtiendo aquel último término, como Eterno Retorno de lo Diferente. ${ }^{29}$ De tal manera, el análisis de los términos que conforman la noción será suficiente para darnos una idea aproximada del funcionamiento de esta síntesis.

Con respecto al tercer término del sintagma, lo que se afirma es que el objeto del retorno, aquello de lo cual éste se predica, es lo diferente: "El sujeto del eterno retorno no es lo mismo, sino lo diferente, ni lo semejante, sino lo disímil, ni el Uno, sino lo múltiple". ${ }^{30} \mathrm{El}$ segundo término, por su parte, adquiere sentido al tornar equivalentes al retorno y la repetición, de manera tal que la repetición tenga como objeto a lo diferente, otorgándole a ésta una consistencia propia:

${ }^{28}$ Según la lectura que hace el propio Deleuze, la noción de Eterno Retorno se encuentra en un estado implícito o larvario en la obra de Nietzsche, que no pudo desarrollarla por razones meramente empíricas: la enfermedad le impidió escribir la cuarta parte del Zaratustra donde había proyectado hacerlo. Por nuestra parte, no nos interesa demasiado juzgar hasta qué punto Deleuze es fiel o no al legado nietzscheano. ${ }^{29}$ Deleuze no es en absoluto ajeno a esta operación, sino que, con la voluntad de seguir a Nietzsche, aclara que aquel "mismo" no es el sujeto del eterno retorno sino su efecto simulado.

${ }^{30}$ DR, p. 195. 
El eterno retorno no hace volver "lo mismo", pero el volver constituye el único Mismo de lo que deviene. Retornar es el devenir-idéntico del devenir mismo. Retornar es pues la única identidad, pero la identidad como potencia segunda, la identidad de la diferencia, lo idéntico que se dice de lo diferente, que gira en torno de lo diferente. Semejante identidad, producida por la diferencia, es determinada como "repetición". ${ }^{31}$

La relación entre la diferencia y la repetición queda entonces definida del siguiente modo: "Si la diferencia es el en-sí, le repetición en el eterno retorno es el para-sí de la diferencia". ${ }^{32}$ De tal manera, es bajo esta noción que la diferencia y la repetición encuentran su afinidad indisoluble, constituyendo la fórmula del devenir: sólo lo que difiere se repite, sólo se repite lo que difiere. Pero desde Nietasche y la filosofía, ${ }^{33}$ donde por primera vez se plantea la noción del eterno retorno, Deleuze insiste con que no alcanza con afirmar el ser del devenir, sino que hay que afirmar que el devenir es el Ser. Por lo tanto, el primer término del sintagma, al establecer la eternidad del retorno, da cuenta de la modalidad necesaria del devenir, lo que lo transforma en un principio o una ley - "el eterno retorno se revela sin duda como la 'ley' sin fondo"- $-{ }^{34}$ El Ser mismo, entonces, en tanto devenir, se comprende como un principio de selección ontológico: sólo es lo que se repite o retorna, pero sólo se repite o retorna lo que difiere. Es por ello que Deleuze dota al eterno retorno de una fuerza centrífuga, que expulsa todo lo que es idéntico, todo lo que no puede cambiar, a la vez que selecciona todo aquello que puede transformarse, volviendo como otro con respecto a su ser inicial. En tanto principio, pues, el eterno retorno invierte la noción clásica de fundamento. Si éste se consideraba como un principio de selección ontológico cuya operación era dotar de semejanza, gracias a su propia identidad, a las materias desemejantes que lo atravesaran, el eterno retorno tiene como operación, por el contrario, la producción de lo diferente a partir lo idéntico o lo semejante, razón por la cual su operación desfonda todo posible fundamento: "Así, el fundamento fue superado hacia un sin fondo, universal desfondamiento que gira en sí mismo y no

\footnotetext{
${ }^{31}$ DR, p. 79.

32 DR, p. 195.

${ }^{33}$ Deleuze G., Nietzsche y la filosofia, trad. Carmen Artal, Barcelona, Anagrama, 1971.

${ }^{34}$ DR, p. 194.
} 
hace volver más que el porvenir". ${ }^{35} \mathrm{El}$ eterno retorno es, pues, la ley del sin fondo, el principio de desfondamiento universal, y el motor de la producción de lo nuevo gracias a su potencia máxima de destrucción; nos da la última palabra sobre la diferencia y la repetición, el punto en el cual éstas se transforman en nociones propiamente ontológicas, y la base a partir de la cual, por lo tanto, habrá que construir la filosofía de la diferencia.

En resumen, es la facultad del pensamiento puro la que, al enfrentarse a su propia fisura, puede acceder a la forma pura del tiempo. $\mathrm{Y}$ es esta forma pura del tiempo la que nos conduce a la tercera repetición, la del devenir, que define el movimiento ontológico del eterno retorno. Así pues, si los cogitanda definen el objeto propio del pensamiento, es decir, aquello que es impensable pero que sin embargo exige ser pensado, los cogitanda deben ser determinados como los elementos del eterno retorno, como las preguntas o imperativos del Ser:

Si los imperativos del Ser tienen una relación con el Yo, la tienen con un Yo fisurado, cuya fisura desplazan y reconstituyen cada vez según el orden del tiempo. Por consiguiente, los imperativos forman los cogitanda del pensamiento puro, las diferenciales del pensamiento; a la vez lo que no puede ser pensado, pero que debe serlo, y no puede sino serlo desde el punto de vista del ejercicio trascendente. $\mathrm{Y}$ las preguntas son esos pensamientos puros de los cogitanda. ${ }^{36}$

En conclusión, si se lo compara con el cogito cartesiano, el cogito para un yo disuelto deleuziano parece un cogito paradójico: no sirve ni para garantizar un Yo idéntico ni para proveer un fundamento objetivo que funcione como soporte del edificio del conocimiento. Por el contrario, tal como hemos visto, el cogito deleuziano nos conduce, en cada uno de sus momentos, más acá o más allá del sujeto idéntico, ya sea el más acá de los sujetos larvarios y las almas contemplativas que funcionan como campo receptivo, el más allá intersubjetivo del pasado en sí, o el mundo impersonal abierto por el eterno retorno. De hecho, es este último, como fin del proceso y resultado, el que constituye el verdadero mundo a-subjetivo que, tal como lo confiesa en el Prefacio, pretende iluminar Deleuze:

35 DR, p. 149.

${ }^{36}$ DR, p. 301. 
Creemos en un mundo donde las individuaciones son impersonales, y las singularidades, preindividuales: el esplendor del "SE". (...) Lo que este libro hubiera debido mostrar es, entonces, la aproximación de una coherencia no es ni la nuestra, la del hombre, ni la de Dios o del mundo. En este sentido hubiera debido ser un libro apocalíptico (el tercer tiempo en la serie del tiempo). ${ }^{37}$

Pero a pesar de aquella contraposición con el cogito cartesiano, es sin embargo un cogito en la medida en que nos presenta un movimiento necesario que, si bien nos conduce a un desfondamiento universal, nos muestra cómo la experiencia de ese desfondamiento nos da la última palabra de lo que significa pensar y nos permite, a partir de aquella constatación, reconstruir otra vez el mundo, aunque este nuevo mundo sea el mundo a-subjetivo de las individuaciones impersonales y de las singularidades preindividuales.

\section{Bibliografía}

- Beaufret, Jean, Hölderlin et Sophocle, Brionne, G. Monfort, 1983.

- Deleuze Gilles, Nietzsche y la filosofía, trad. Carmen Artal, Barcelona, Anagrama, 1971.

- Deleuze, Gilles, Diferencia y repetición, trad. María Silvia Delpy y Hugo Beccacece, Buenos Aires, Amorrortu, 2002.

- Williams, James, Gilles Deleuze's Difference and repetition: a critical introduction and guide, Edinburgh, Edinburgh University Press, 2003.

Recibido: 04/2019. Aceptado: 08/2019

${ }^{37}$ DR, p. 18. 